STRUCTURAL BIOLOGY
Keywords: book reviews; biomolecular smallangle scattering.

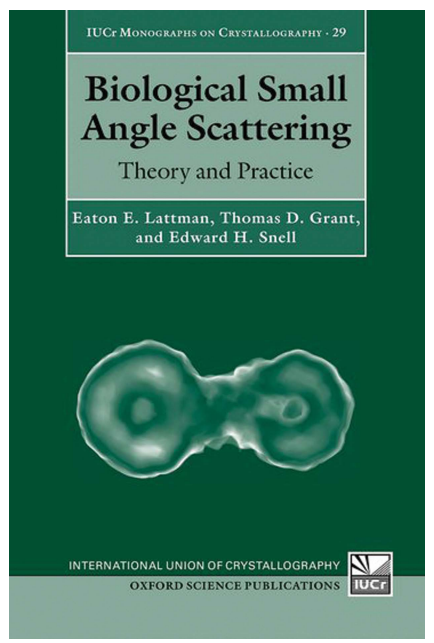

(C) 2019 International Union of Crystallography

\section{Biological Small-Angle Scattering: Theory and Practice. By Eaton E. Lattman, Thomas D. Grant and Edward H. Snell. Oxford University Press, 2018. Pp. 288. Price GBP 65.00, hardback, ISBN 9780199670871.}

\author{
Jill Trewhella*
}

School of Life and Environmental Sciences, The University of Sydney, Sydney, Australia. *Correspondence e-mail: jill.trewhella@sydney.edu.au

This monograph on biomolecular small-angle scattering is divided into four parts: a brief introduction that provides an overview of the technique and what can be learned from its application to biological systems, followed by a treatment of the underlying theory, then practical aspects and finally applications. The text is well written and easy to read, with each chapter concluding with a helpful summary. Overall, the book provides a description of biomolecular small-angle scattering and its applications that complements other texts and, when used in combination with established quality reference texts that are well referenced in this new contribution, would be useful for both the novice and expert scatterer.

The introductory chapter is succinct and sets the tone for the rest of the book. For the theory chapters that follow, a stated goal was to provide an 'intuitive heuristic approach' to scattering theory rather than a 'rigorously formal' one. This challenging goal is one in which the authors have been successful, particularly in Chapter 2 covering Theoretical Background. For the reader learning about the technique for the first time, the text here is generally more understandable and accessible compared with more formal, typically physics-oriented texts. For the expert reader, some of the descriptions provide useful ways to explain phenomena in a teaching context. The explanations are particularly 'crystallographer friendly', often starting with formalisms that are commonly used in crystallography. This approach is useful in that a significant portion of the growth in biological small-angle scattering comes from practicing crystallographers. In this regard, the discussion of resolution in the context of solution scattering is excellent and important given the general confusion around resolution for solution scattering. There is also an excellent discussion of the limitations of ab initio shape modelling. A small criticism is that the problem of uniqueness of three-dimensional structural modelling based on solution scattering data comes up for the first time in the middle of Chapter 2; this would have ideally been addressed earlier in the introductory Chapter 1, given that this issue is central to having a balanced understanding of the strengths and limitations of biomolecular solution scattering.

Chapter 3 moves on to a useful and comprehensive description of the quantities that can be derived from scattering data. Here the authors begin reverting to descriptions in terms of the scattering from electrons, where it would have been of value to write in more general terms of the scattering of X-rays or neutrons by atoms, given that the authors' intention was to consider both SAXS and SANS. The absence of any definitions of units makes the book of limited value as a practical guide by itself, although the references in Part 3 can make up for this omission. It is pertinent to note here also that there are a few errors in the first printing, most notably in equations 3.3 and 3.4 deriving molecular weight from the intensity at the origin: Avogadro's number should be in the denominator in equation 3.3 and in the numerator for equation 3.4. The authors are working with the publisher to correct these equations and other typographical errors in the second printing and in the electronic edition. An erratum for the first printing will be available.

While Chapter 4 is titled Shape Reconstruction, it is actually a more comprehensive discussion of three-dimensional modelling methods, including atomistic rigid-body and ensemble modelling, and it appropriately balances the strengths and limitations of different modelling approaches. The authors describe the 'method of choice' for comparing model fits in terms of $\chi^{2}$, which is the traditional measure. However, the most 
recent work towards community-agreed recommendations for model validation from small-angle scattering data has focused on complementary methods for evaluating model fits that address the limitations of $\chi^{2}$ as a global parameter of fit that does not identify potentially significant localized regions of misfit and is highly influenced by the magnitude of the propagated errors. A fuller discussion of these issues and developments is an unfortunate omission for a reference text at this stage.

Part 3 dealing with the practical aspects of small-angle scattering encompasses Chapters 5-11, with Chapters 5-10 covering what to do before and during a small-angle scattering experiment, data collection and processing, instrumental and experimental considerations and approaches. Chapter 7 on data collection and processing stands out as particularly excellent and useful, although it would have been good to include here some discussion of the importance of accurate error propagation, given how influential errors are in evaluating the quality of model fits to data, for example using $\chi^{2}$. Chapter 9 on instruments is quite idiosyncratic, providing a lot of different kinds of detail on a selected set of instruments. Given that it is, as is acknowledged, neither comprehensive nor systematic, the level of detail is nevertheless potentially misleading about what is available where. A useful addition would have been a matrix of considerations for the planned experiment and general instrument characteristics to consider in making a choice of what kind of instrument/beamline might be required. Overall, Chapters 5-10 are generally well referenced and as such provide a useful introduction and grounding in ideas and concepts for the reader, who can then depend upon specific recommended references for more complete and detailed practical guides as they approach specific experiments.

The final Chapter 11 in Part 3 is explicitly about SANS and is a somewhat disappointing introduction to this powerful technique. The description of the analysis of SANS data is incomplete, for example with reference to the extraction of structural parameters for the components of complexes. Curiously, equation 12.1 that applies most commonly to SANS contrast-variation analysis is introduced in the much less common X-ray contrast-variation example in Part 4. Also, the authors have chosen examples to highlight in Chapter 11 that ignore significant applications and recent landmark experiments, although again the omissions are partly helped by the SANS contrast-variation section (12.1.1) in Part 4. Overall, the treatment of SANS is weak compared with how SAXS is covered, which is perhaps simply a consequence of the facts that there are far fewer facilities for SANS and that the experiments are more demanding technically and in terms of samples. The authors do provide a basic introduction, but for those keen to learn about this technique and its applications, other references would be better.

The final Part 4 includes Chapters 12-14 that present a set of applications past, present and future. The selected examples provide an interesting perspective that is particular to these authors on where the field has been and where it is going. Chapter 12 draws on the authors' selection of examples that highlight the kinds of questions that small-angle scattering has answered from a structural perspective. Chapter 13 considers time-resolved studies of dynamic processes using highintensity synchrotrons and XFELS. Finally, Chapter 14 (Pushing the Envelope) is an expansive description of a recent publication by one of the authors of a new method proposed for retrieving electron-density distributions directly from solution scattering data. This idea is certainly intriguing, but is also quite untested in the broader community. As such, elevating it to a whole chapter in what aims to be a reference book is perhaps not advisable, although there are qualifiers acknowledging that the technique is in its 'infancy'.

The book closes with the essential resources that make it most useful as a reference text: definitions of acronyms, a glossary of terms, definitions of major variables and a comprehensive alphabetized reference list with titles, and a thorough index. There are also useful Appendices covering the structure-factor equation and troubleshooting your experiment. Appendix A.2 notes recent work in the community to establish publication guidelines that aim to increase the reliability and impact of biomolecular small-angle scattering in structural biology, where, as in this book overall, a continued bright future is envisioned for the field. 